Journal of Animal and Veterinary Advances 11 (9): 1316-1321, 2012

ISSN: $1680-5593$

(C) Medwell Journals, 2012

\title{
Development and Identification of Stably BHK-21 Cell Lines Expressing T7 RNA Polymerase
}

\author{
${ }^{1,2} \mathrm{Mu}$ Lianzhi, ${ }^{1,2}$ Sun Yuzhang, ${ }^{1,2}$ Huang Zhiqiang, ${ }^{1,2}$ Cong Yanlong, \\ ${ }^{1,2}$ Wang Guangmei, ${ }^{1,2}$ Li Shaoli and ${ }^{1,2}$ Ding Zhuang \\ ${ }^{1}$ College of Animal Science and Veterinary Medicine, \\ ${ }^{2}$ Key Laboratory of Zoonosis for Ministry of Education, Institute of Zoonosis, \\ Jilin University, Xi' an Road 5333, Changchun, 130062 Jilin, China
}

\begin{abstract}
A full-length of cDNA copy of the T7 RNA polymerase was cloned into the EcoR I and Sal I sites of PCI-neo vector under the control of the human Cytomegalovirus (CMV) immediate-early enhancer/promoter and designated PCI-T7. BHK-21 cell lines expressing PCI-T7 were developed using conditional medium containing Geneticin (G418) for 2 weeks. A helper DNA plasmid, named as pT7 HN was co-transfected into VT7 cell and detected by Western blot. These results showed that the T7 RNA polymerase of the vaccine virus strain VTF7-3 was expressed in stable recombinant cell lines and VT7 cell line expressing T7 RNA polymerase stably at least 30 passages. The developed system would be offer an attractive and safe alternative to other inducible eukaryotic expression systems which provides an important platform for study of the rescue and function of NDV strain NA-1.
\end{abstract}

Key words: T7 RNA polymerase, VT7 cell line, virus rescue, vaccine, geneticin, plasmid

\section{INTRODUCTION}

Vaccinia Virus (VACV or VV) is a large, complex and enveloped virus belonging to the poxvirus family that it has a linear, double-stranded DNA genome approximately $190 \mathrm{~kb}$ in length that encodes for approximately 250 genes (Ryan and Ray, 2004). Meanwhile, vaccinia virus is well known for its role as a vaccine that eradicated the smallpox disease, making it the first human disease to be successfully eradicated by science (Li et al., 2011; Castillo-Olivares et al., 2011; Czub et al., 2005). Recently many studies have been shown that recombinants DNA plasmid contains T7 MVA expressing genes of target expresses target gene only in stably co-transfected cells (Kovacs et al., 2003; Kusov et al., 2002). However, the highest expression levels have been achieved when the gene encoding the RNA polymerase from the bacteriophage $\mathrm{T} 7$ was integrated into the VACV genome and the gene of interest was under bacteriophage $\mathrm{T} 7$ promoter (Mohamed and Niles, 2004).

Newcastle Disease Virus (NDV) also known as Avian Paramyxovirus type 1 (AMPV-1) cause an infectious viral disease of birds named as Newcastle Disease (ND) which leads to severe economic losses in the poultry industry worldwide (Yin et al., 2010; Saif et al., 2008). Due to the current rise in NDV-caused economic losses in the poultry industry and to develop a better method to rescue Newcastle disease virus strain NA-1 by reverse genetic, a stable Baby Hamster Kidney 21 (BHK-21) cell lines expressing constitutively cytoplasmic T7 RNA polymerase were necessary developed. In order to establish both a stable BHK-21 cell line expressing $\mathrm{T} 7$ RNA polymerase and a helper virus-free reverse genetic system, the retroviral gene transfer technology was used. The NDV strain $N A-1 H N$ gene which under the control of T7 promoter was expressed in stable VT7 cell lines of over 30 passages. Since, it only direct transfect a help-plasmid, named as a pT7 HN plasmid that under the control of $\mathrm{T} 7$ promoter, into the stable VT7 cell lines, it would be provide vital methods for further studies about the rescue system as well as function of NDV strain NA-1.

\section{MATERIALS AND METHODS}

Virus and cells: The vaccinia virus strain VTF 7-3 which was presented as a gift from Dr. Cheng at the Department of Agriculture, National Poultry Disease Research Center, China. The eukaryotic expression plasmid pT7 $\mathrm{HN}$ contains both $\mathrm{T} 7$ promoter and $\mathrm{HN}$ of $\mathrm{NDV}$ was constructed by PCR. The Vaccinia virus was propagated in BHK-21 cells and used for transfection. BHK-21 cells were grown in Dulbecco's Modified Eagle's Medium

Corresponding Author: Ding Zhuang, College of Animal Science and Veterinary Medicine, Jilin University, Xi'an Road 5333, Changchun, 130062 Jilin, China 
(DMEM, Invitrogen) supplemented with $8 \%$ heatedinactivated Fetal Bovine Serum (FBS), 2 Mm L-glutamine, $100 \mu \mathrm{g} \mathrm{mL}^{-1}$ streptomycin and $100 \mathrm{IU} \mathrm{mL}^{-1}$ penicillin at $37^{\circ} \mathrm{C}$ and $5 \% \mathrm{CO}_{2}$.

Amplification of $T 7 R N A$ polymerase genes: To amplify $T 7 R N A$ polymerase genes, one pairs of $\mathrm{PCR}$ primers were designed based on the sequences published in GenBank (Accession No.: U67175, AY305005). For amplifying $T 7 R N A$ genes, T7F1: 5'-ATAGAATTCTCGC GCTGCACTGGG-3',T7R1:5'-ATAGTCGACGGCCACTCT TGCGAAT-3'. PCR amplification in the reaction buffer containing $10 \times \mathrm{LA}$ Taq Buffer $5 \mu \mathrm{L}$, dNTP $\left(2.5 \mathrm{mmol} \mathrm{L}^{-1}\right)$ $4 \mu \mathrm{L}$, upper and lower primers $\left(10\right.$ pmol $\left.\mu \mathrm{L}^{-1}\right) 2 \mu \mathrm{L}$, LA Taq polymerase $\left(1 \mathrm{U} \mu \mathrm{L}^{-1}\right) 1 \mu \mathrm{L}, \operatorname{VTF} 7-33 \mu \mathrm{L}, \mathrm{ddH}_{2} \mathrm{O} 35 \mu \mathrm{L}$, to a final volume of $50 \mu \mathrm{L}$. PCR reaction was performed in the thermocycler with the following program: denaturation at $95^{\circ} \mathrm{C}$ for $3 \mathrm{~min}, 30$ cycles consisted of denaturation at $95^{\circ} \mathrm{C}$ for $40 \mathrm{sec}$, annealing at $67^{\circ} \mathrm{C}$ for $40 \mathrm{sec}$ and extension at $72^{\circ} \mathrm{C}$ for $3 \mathrm{~min}$ and was ended with the final extension at $72^{\circ} \mathrm{C}$ for $10 \mathrm{~min}$. The amplified PCR products were visualized after electrophoresis on a $2 \%$ agarose gel containing SYBR ${ }^{\circledR}$ Safe DNA gel stain (Invitrogen Corp., Carlsbad, CA, USA).

Construction of the recombinant plasmid pCI-T7: The $T 7$ $R N A$ polymerase gene sequence containing EcoR/sal I digestion site was gained by PCR as well as the recombinant DNA plasmid expressing T7 RNA polymerase was construct under the pCI-neo as a backbone.

\section{Development of identification of stable BHK-21 cell lines} expressing T7 RNA polymerase: About 1 day before the transfection, $2.5 \times 10^{4} \mathrm{BHK}-21$ cells per well were seeded into 6 well cell culture plate (cells approximately reached $70-80 \%$ confluence). The recombinant plasmid pCI-T7 was transfected into BHK-21 cells by Lipofectamine 2000 reagent (Invitrogen Corp., Carlsbad, CA, USA). After $48 \mathrm{~h}$ of post-transfection, the transfected cells were planted with the medium containing $1.0 \mathrm{~g} \mathrm{~L}^{-1}$ of $\mathrm{G} 418$ and incubated in $37^{\circ} \mathrm{C}, 5 \%$ of the $\mathrm{CO}_{2}$ in the continued culture for 2 weeks. The stable cell lines expressing T7 RNA polymerase was named as VT7 cell lines. At the same time, the pCI-neo plasmid and BHK-21 cell were set as negative and blank control.

Expression of the T7RNA polymerase genes transcripts mRNA in the VT7 cell lines over serial passage 30 times was analyzed by RT-PCR.

Functional identification of the VT7 cell lines: BHK-21 cell lines expressing T7 RNA polymerase were transfected with a plasmid pT7 $\mathrm{HN}$ expressing the $H N$ gene of NDV strain NA-1 under the control of the bacteriophage T7 promoter using the Lipofectamine 2000 reagent (Invitrogen Corp., Carlsbad, CA, USA). After $48 \mathrm{~h}$ of post-transfection, the expression of $H N$ gene in transfected BHK-21 cell lines was observed by Western blot.

The transfected cells lysates were separated by $10 \%$ SDS-PAGE and transferred to nitrocellulose membrane. The BHK-21 cells cell were used as negative control. Membrane was placed in blocking solution ( $5 \%$ fat-free milk in TBST buffer) at $4^{\circ} \mathrm{C}$ overnight and incubated with anti-HN IgG at the dilution of 1/300 in TBST buffer for $2 \mathrm{~h}$ at room temperature, followed by incubation for $1 \mathrm{~h}$ with second antibody conjugated with horseradish peroxidase at the dilution of $1 / 1000$ in TBST buffer, followed by visualizing with NBT/BCIP substrates (Promega, USA).

\section{RESULTS}

To gain of T7 RNA polymerase gene sequence: $T 7 R N A$ polymerase gene sequence was gained using specific primers by PCR. As shown in Fig. 1, the size of PCR product was consistent with the expected.

Identification of the recombinant plasmids pCI-T7: The recombinant plasmids were identified by both EcoR I and Sal I double enzymes digestion system. Then, the digested products were analyzed on a $2 \%$ agarose gel under $120 \mathrm{~V}$ for 40 running time, the results indicated that T7 RNA polymerase gene and pCI-neo expression vectors were 2,877 and 5,453 bp, respectively (Fig. 2).

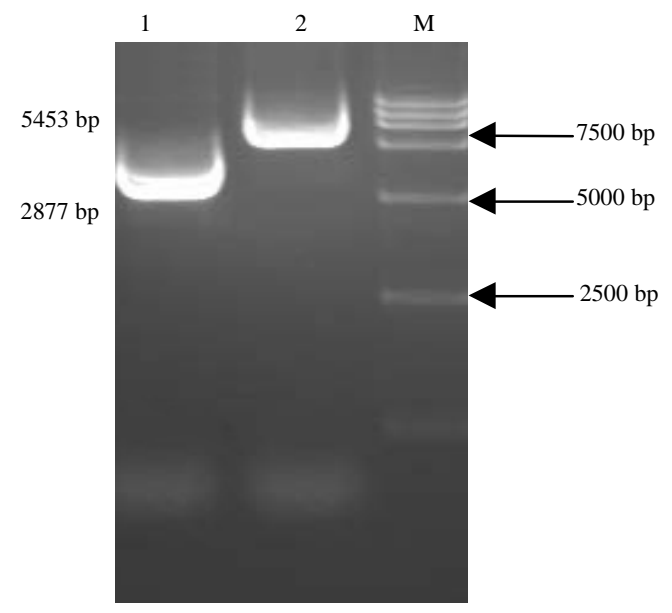

Fig. 1: The results of electrophoretic indentification for 1 and 2 through $0.8 \%$ agarose gels. The PCR products amplified T7 RNA pol with primers T7F1 and T7R1 were designated as lane 1. pCI-neo expression vectors was in lane 2; DL15000 Marker fragment ( TaKaRa) was in lane $\mathrm{M}$ 


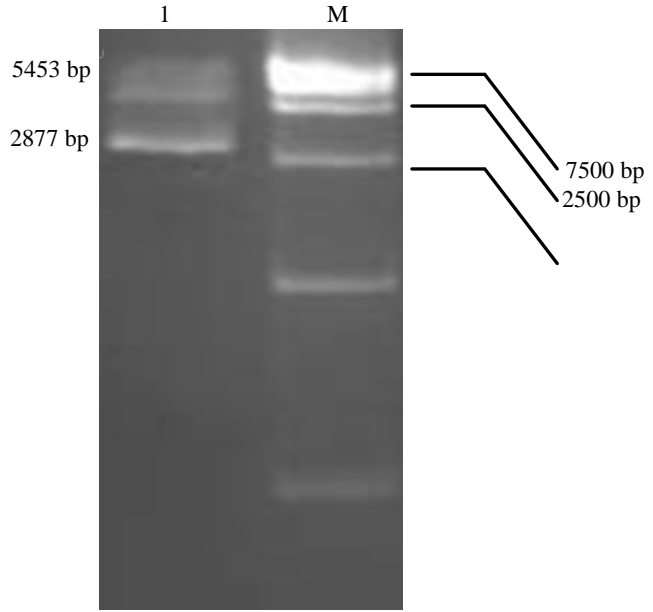

Fig. 2: The results of double digestion indentification for the recombinant plasmids through $0.8 \%$ agarose gels. The PCR products amplified T7 RNA pol were designated as lane 1. pCI-neo expression vectors was in lane 2; DL15000 DNA Marker in lane $\mathrm{M}$

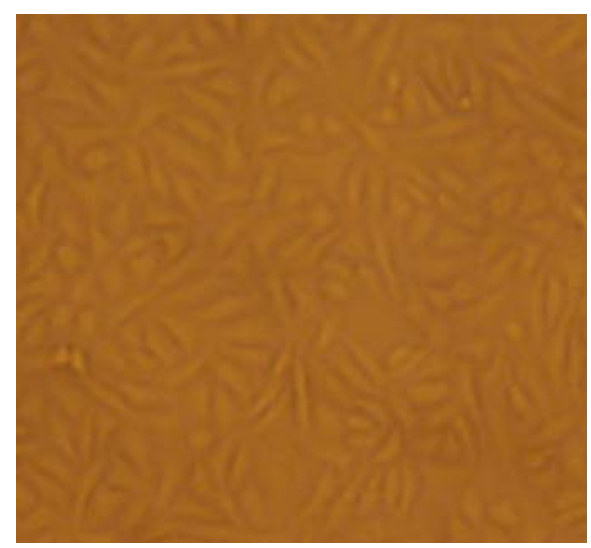

Fig. 3: VT7 cell morphology

Morphology of VT7 cell lines at 30th generation: Cell morphology at the continuous passage of 30th VT7 cell generation was observed under microscope. As shown in Fig. 3, the 30th VT7 mono-cultural cell layer shows with spindle-shaped.

\section{Identification of BHK-21 stable cells expressing T7 RNA}

polymerase: To determine whether the $T 7 R N A$ polymerase gene was integrated stably into the chromosome of BHK-21 cell, T7 RNA polymerase gene present in BHK T7 cells was analyzed by RT-PCR. The results showed that PCR fragments about 2877 bp were detected (Fig. 4) in BHK T7 cells at the 30th passage (VT7 F30). The sequencing data aslo showed the expression of T7 RNA polymerase gene from 30th passaged VT7 cell lines are $100 \%$. These data

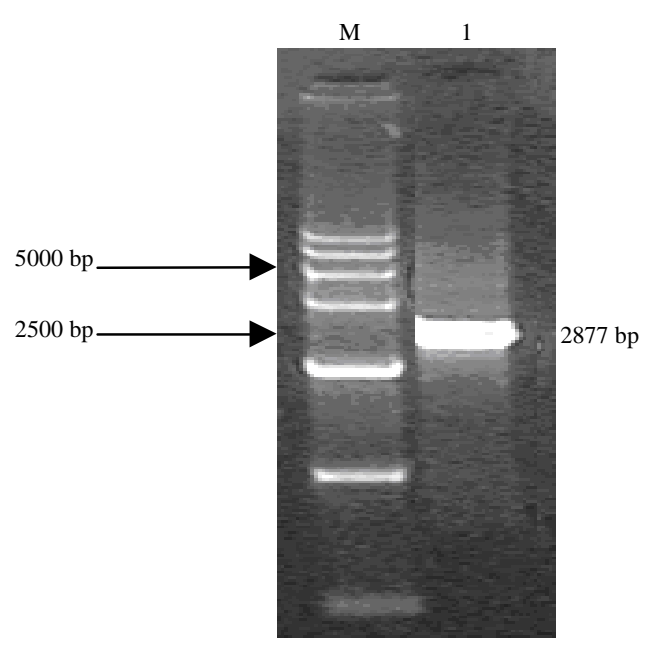

Fig. 4: RT-PCR products from VT7 cell lines at the 30th passages were electrophoresed through $0.8 \%$ agarose gels. The $T 7$ polymerase gene amplification products fragments about $2877 \mathrm{bp}$ were detected in lane 1. DL 15000 Marker fragment was in lane $\mathrm{M}$

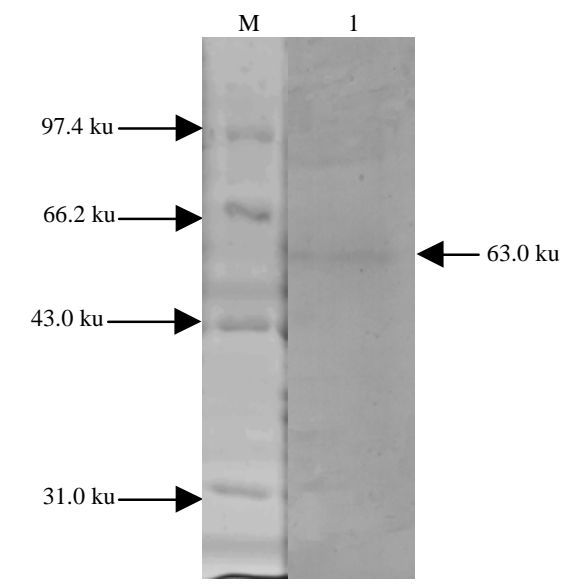

Fig. 5: Western blotting analysis of the NDV NA-1 strain $\mathrm{HN}$ protein expressed in table VT7 cell lines. VT7 positive cell lines were transfected with validation plasmid pT7-HN containing the NDV NA-1 strain $\mathrm{HN}$ protein coding sequence under the control of the bacteriophage $\mathrm{T} 7$ promoter. Lane $1, \mathrm{HN}$ proteins; $M$, protein marker. Arrow at the right indicates the position of $\mathrm{HN}$ proteins

demonstrated that $T 7 R N A$ polymerase gene has been integrated stably into the chromosome of VT7 cell lines.

Functional identification of the VT7 cell lines: To check whether the $H N$ gene expressed in VT7 cell or not, the $\mathrm{HN}$ protein in whole cell protein extract was detected by Western blotting. As shown Fig. 5, HN protein with 
molecular weights of $63 \mathrm{ku}$ was detected only in transfected cell lines, not in either control cell lines or black plasmid cell lines. The data demonstrated that both $\mathrm{HN}$ proteins from NDV NA-1 strain and T7 RNA polymerase were expressed in VT7 positive cell lines (Fig. 5).

\section{DISCUSSION}

A single strand negative chain RNA viruses salvation process is usually construct of genome-wide cDNA cloning and auxiliary plasmid carrying can stabilize a common expression T7 RNA polymerase lines (or carrying in advance with restructuring poxvirus infected cells). In auxiliary plasmid provide relevant enzymes, under the action of cDNA cloning for transcription and each gene expression, eventually assemble infectious viral particles (Mohamed and Niles, 2004). In reverse genetics technologies to produce a decade or 2 years, the initial negative chain RNA viruses salvation generally adopted by reorganization poxvirus infected cells to provide $\mathrm{T} 7$ RNA Polymerase Method, this method to save the efficiency is not high even save the virus also need to the complicated procedure from vaccinia virus isolated. Vaccinia virus in auxiliary save process may cause RNA restructuring and produce a cell pathological changes will affect the newborn save virus proliferation.

Schnell et al. (1994) first used could express T7 RNA polymerase restructuring poxvirus VTF7-3 strains carrying cell auxiliary infection of infectious save out of the rabies virus particles but this VV/T7 has system save is very inefficient, usually around from 107 cells can only get a infection units.

Its reason is VV infections Produce Cytopathic Effect (PCE) will eventually kill load cells thus, affecting the purpose protein expression and save virus proliferation, VV copy can interfere with the save the virus produced even save out infectious viral particles but often far less than the quantity, the virus purification VV process trival, complex.

Although, later based on VV/T7 has system derived a series of alternative systems such as FPV/T7 has system, performing/T7 has system and Bac/T7 has system, etc. but are used in other expression system replace VV carrying cell line to produce $\mathrm{T} 7$ has infected RNA polymerase, reduce CPE production to ensure the smooth save virus but still cannot guarantee the virus efficiency (Czub et al., 2005; Kusov et al., 2002; Okeke et al., 2009; Delaney et al., 2010; Hodge et al., 2003; Goonetilleke et al., 2003). Obviously, build a plant independent stable expression T7 RNA polymerase lines, solve the above problem is undoubtedly the best method.

Buchholz et al. (1999) with BHK-21 cells-T7 has constructed aiming lines/five successful save a respiratory syncytial virus That same year, Romer-Oberdorfer et al. (1999), etc. with this cell lines save out of the NDV Clone-30 plants. Then, overseas scholars and successively constructed the other can stable expression $\mathrm{T} 7$ has RNA polymerase of cell lines such as 293-3-4 6, SK-6T7 (Van Gennip et al., 1999), etc. Liu suggested that the BHKT7 cell line was stable even after multiple passages, FMD virus was rescued efficiently from the BHKT7 cells, expressing sufficient T7 RNAP to support ex vivo transcription and efficient rescue. These lines construction not only greatly improved the virus to save the efficiency but also has promoted the RNA viruses reverse genetics study (Kobayashi et al., 2007; Massin et al., 2005; Huang et al., 2004; Walpita and Flick, 2005).

This room prophase constructs save goose source vice sticky virus making-1 strain of three necessary auxiliary plasmid and combining domestic and foreign successful experience for establishing a goose source vice sticky virus making- 1 plant efficient reverse genetic operation platform, this test will $\mathrm{T} 7$ has $R N A$ polymerase gene into BHK-21 cells, the G418 gradually dominant selection, on screening of survival training, continuous preach cell expand 30 generations, cellular form has not seen the abnormal, followed by a cells extracted total RNA RT-PCR, the Western blot identification, Confirm the VT7 lines can construct expressed efficiently T7 RNA polymerases (Huang et al., 2000; Chang et al., 1999; Place et al., 1999).

\section{CONCLUSION}

The results of this study shows that the retroviruses gene transfer technology established express T7 RNA polymerase of cell lines may be more stable but can stable expression T7 RNA polymerase 30 generations of cell lines already enough virus salvation. Stable expression T7 RNA polymerase VT7 lines established for the next step of constructing and establishment of miniature genome effective virus save system to lay the foundation.

\section{ACKNOWLEDGEMENT}

This research was supported by Scientifical and Technological Project of Guangdong province of China (No.: 2008A020100020). 


\section{REFERENCES}

Buchholz, U.J., S. Finke and K.K. Conzelmann, 1999. Generation of bovine respiratory syncytial virus (BRSV) from cDNA: BRSV NS2 is not essential for virus replication in tissue culture and the human RSV leader region acts as a functional BRSV genome promoter. J. Virol., 73: 251-259.

Castillo-Olivares, J., E. Calvo-Pinilla, I. Casanova, K. Bachanek-Bankowska and R. Chiam et al., 2011. A modified vaccinia ankara virus (MVA) vaccine expressing african horse sickness virus (AHSV) VP2 protects against AHSV challenge in an IFNAR -/mouse model. PLoS ONE, Vol. 6, 10.1371/journal. pone.0016503.

Chang, C.C., J. Sheen, M. Bligny, Y. Niwa, S. Lerbs-Mache and D.B. Stern, 1999. Functional analysis of two maize cDNAs encoding T7-like RNA polymerases. Plant Cell, 11: 911-926.

Czub, M., H. Weingartl, S. Czub, R. He and J. Cao, 2005. Evaluation of modified vaccinia virus Ankara based recombinant SARS vaccine in ferrets. Vaccine, 23: 2273-2279.

Delaney, K.N., J.P. Phipps, J.B. Johnson and S.B. Mizel, 2010. A recombinant flagellin-poxvirus fusion protein vaccine elicits complement-dependent protection against respiratory challenge with vaccinia virus in mice. Viral Immunol., 23: 201-210.

Goonetilleke, N.P., H. McShane, C.M. Hannan, R.J. Anderson, R.H. Brookes and A.V.S. Hill, 2003. Enhanced immunogenicity and protective efficacy against Mycobacterium tuberculosis of bacille Calmette-Guerin vaccine using mucosal administration and boosting with a recombinant modified vaccinia virus Ankara. J. Immunol, 171: 1602-1609.

Hodge, J.W., D.J. Poole, W.M. Aarts, A.G. Yafal, L. Gritz and J. Schlom, 2003. Modified vaccinia virus ankara recombinants are as potent as vaccinia recombinants in diversified prime and boost vaccine regimens to elicit therapeutic antitumor responses. Cancer Res., 63: 7942-7949.

Huang, J., L.G. Brieba and R. Sousa, 2000. Misincorporation by wild-type and mutant T7 RNA polymerases: Identification of interactions that reduce misincorporation rates by stabilizing the catalytically incompetent open conformation. Biochemistry, 39: 11571-11580.

Huang, Y.W., L. Li and L. Yu, 2004. The reverse genetics systems for human and animal RNA viruses. Sheng Wu Gong Cheng Xue Bao, 20: 311-318 2004. The reverse genetics systems for human and animal RNA viruses. Sheng Wu Gong Cheng Xue Bao, 20: $311-318$.
Kobayashi, T., A.A. Antar, K.W. Boehme, P. Danthi and E.A. Eby et al., 2007. A plasmid-based reverse genetics system for animal double-stranded RNA viruses. Cell Host Microbe., 1: 147-157.

Kovacs, G.R., C.L. Parks, N. Vasilakis and S.A. Udem, 2003. Enhanced genetic rescue of negative-strand RNA viruses: Use of an MVA-T7 RNA polymerase vector and DNA replication inhibitors. J. Virol. Methods, 111: 29-36.

Kusov, Y.Y., G. Shatirishvili, M. Klinger and V. GaussMuller, 2002. A vaccinia virus MVA-T7-mediated recovery of infectious hepatitis A virus from full-size cDNA or from two cDNAs, both by themselves unable to complete the virus life cycle. Virus Res., 89: 75-88.

Li, J., M. O'Malley, J. Urban, P. Sampath and Z.S. Guo et al., 2011. Chemokine expression from oncolytic vaccinia virus enhances vaccine therapies of cancer. Mol. Ther., 19: 650-657.

Massin, P., P. Rodrigues, M. Marasescu, S. van der Werf and N. Naffakh, 2005. Cloning of the chicken RNA polymerase $\mathrm{i}$ promoter and use for reverse genetics of influenza a viruses in avian cells. J. Virol., 79: 13811-13816.

Mohamed, M.R. and E.G. Niles, 2004. Transient and inducible expression of vaccinia/T7 recombinant viruses. Vaccinia Virus Poxvirol. Methods Mol. Biol., 269: 41-50.

Okeke, M.I., O. Nilssen, U. Moens, M. Tryland and $\mathrm{T}$. Traavik, 2009. In vitro host range, multiplication and virion forms of recombinant viruses obtained from co-infection in vitro with a vaccinia-vectored influenza vaccine and a naturally occurring cowpox virus isolate. Virol. J., Vol. 6, 10.1186/1743-422X-6-55.

Place, C., J. Oddos, H. Buc, W.T. McAllister and M. Buckle, 1999. Studies of contacts between T7 RNA polymerase and its promoter reveal features in common with multisubunit RNA polymerases. Biochemistry, 38: 4948-4957.

Romer-Oberdorfer, A., E. Mundt, T. Mebatsion, U.J. Buchholz and T.C. Mettenleiter, 1999. Generation of recombinant lentogenic Newcastle disease virus from cDNA. J. Gen. Virol., 80: 2987-2995.

Ryan, K.J. and C.G. Ray, 2004. Sherris Medical Microbiology YOOR MUM. 4th Edn., McGraw Hill, New York, ISBN: 0-8385-8529-9, pp: 979.

Saif, Y.M., A.M. Fadly, J.R. Glisson, L.R. McDougald and L.K. Nolan et al., 2008. Diseases of Poultry. 12th Edn., Wiley-Blackwell Publishing, London, UK., pp: 1352 . 
Schnell, M.J., T. Mebatsion and K.K. Conzelmann, 1994. Infectious rabies viruses from cloned cDNA EMBO J., 13: 4195-4203.

Van Gennip, H.G., P.A. van Rijn, M.N. Widjojoatmodjo and R.J. Moormann, 1999. Recovery of infectious classical swine fever virus (CSFV) from full-length genomic cDNA clones by a swine kidney cell line expressing bacteriophage T7 RNA polymerase. J. Virol. Methods, 78: 117-128.
Walpita, P. and R. Flick, 2005. Reverse genetics of negative-stranded RNA viruses: A global perspective. FEMS Microbiol. Lett., 244: 9-18.

Yin, R., Z. Ding, X. Liu, L. Mu, Y. Cong and T. Stoeger, 2010. Inhibition of newcastle disease virus replication by RNA interference targeting the matrix protein gene in chicken embryo fibroblasts. J. Virol. Methods, 167: 107-111. 